\title{
Special Analytical Solutions of the Schrödinger Equation for 2 and 3 Electrons in a Magnetic Field and ad hoc Generalizations to $\mathrm{N}$ particles
}

\author{
by M. Taut \\ Institut für Festkörper und Werkstoff- Forschung Dresden \\ Postfach 270018 \\ 01171 Dresden, Germany \\ email:m.taut@ifw-dresden.de
}

October 25, 2018

\begin{abstract}
We found that the two-dimensional Schrödinger equation for 3 electrons in an homogeneous magnetic field (perpendicular to the plane) and a parabolic scalar confinement potential (frequency $\omega_{0}$ ) has exact analytical solutions in the limit, where the expectation value of the center of mass vector $\mathbf{R}$ is small compared with the average distance between the electrons. These analytical solutions exist only for certain discrete values of the effective frequency $\tilde{\omega}=\sqrt{\omega_{o}^{2}+\left(\frac{\omega_{c}}{2}\right)^{2}}$. Further, for finite external fields, the total angular momenta must be $M_{L}=3 m$ with $m=$ integer, and spins have to be parallel. The analytically solvable states are always cusp states, and take the components of higher Landau levels into account. These special analytical solutions for 3 particles and the exact solutions for 2 particles [13] can be written in an unified form. The first set of solutions reads

$\Phi=\prod_{i<k}\left(\mathbf{r}_{i}-\mathbf{r}_{k}\right)^{m} p_{n, m}\left(\left|\mathbf{r}_{i}-\mathbf{r}_{k}\right|\right) \exp \left(-\frac{1}{2} \tilde{\omega}_{n, m} \sum_{l} \mathbf{r}_{l}^{2}\right)$

where $p_{n, m}(x)$ are certain finite polynomials and $\tilde{\omega}_{n, m}$ is the spectrum
\end{abstract}


of the fields. The pair angular momentum $m$ has to be an odd integer and the integer $n$ defines the number of terms in the polynomials. For infinite solvable fields $\tilde{\omega}_{1}$ there is a second set of the form

$\Phi=\mathcal{A}_{a} \prod_{i<k}\left(\mathbf{r}_{i}-\mathbf{r}_{k}\right)^{m_{i k}} \exp \left(-\frac{1}{2} \tilde{\omega}_{1} \sum_{l} \mathbf{r}_{l}^{2}\right)$

where $\mathcal{A}_{a}$ is the antisymmetrizer and the pair angular momenta $m_{i k}$ can all be different integers. In both cases the first factor is a shorthand with the convention $\mathbf{r}^{m}=r^{|m|} e^{i m \alpha}$. These formulae, when ad hoc generalized to $\mathrm{N}$ coordinates, can be discussed as an ansatz for the wave function of the $\mathrm{N}$-particle system. This ansatz fulfills the following demands: it is exact for two particles and for 3 particles in the limit of small $\mathbf{R}$ and for the solvable external fields, and it is an eigenfuncton of the total orbital angular momentum. The Laughlin functions are special cases of this ansatz for infinite solvable fields and equal pair- angular- momenta.

PACS classification:

8.30.Vw (Quantum dots etc.), 73.30.Hm (Quantum Hall Effect) 


\section{Introduction}

Most work on correlated electron systems in a magnetic field (and a parabolic confinement potential) has been done adopting the following methods: Finite particle number $(N<10)$ and finite field systems are tackled either by numerical expansion of the wave functions in antisymmetrized products of one-particle functions [1]-[6] or analytical ad hoc approaches in the high field limit, where only the lowest Landau level (LLL) contributes [7]-[9]. Other main streams are to use the Chern-Simons transformation [10] [12] and hoping that the transformed wave function can be guessed or approximated more easily, or to use models for the electron- electron interaction [11] [12] (All the above references are mostly reviews). In this paper we are trying another approach: We are looking for analytical solutions for few electron systems and trying to generalize them ad hoc to $\mathrm{N}$ particle systems. We use the genuine Coulombic electron- electron interaction and do not restrict ourselves to the lowest Landau level (LLL). In a previous paper [13 it has been shown that for $N=2$ (with Coulomb interaction between the electrons) there is a 'spectrum' of discrete external field values for which the Schrödinger equation can be solved exactly and analytically. As shown below, these solutions comprise the Laughlin states for $N=2$ as special cases. The questions to be addressed in this paper are the following: Do similar exact solutions also exist for three electrons? If so, does the corresponding field spectrum agree with the spectrum for $N=2$ ? Is there any connection between the discrete field spectrum for solvability and the discrete fields (for given particle density) observed in the Quantum Hall effect? Are the Laughlin states still among these special states as special cases? To answer one of the questions in advance: We did not find exact analytical solutions for $N=3$. However, if we consider the

center of mass coordinate $\mathbf{R}=\frac{1}{3} \sum_{i=1}^{3} \mathbf{r}_{i}$ versus inter-particle distance as an small parameter and expand the Hamiltonian in a multi-pole series, the three- electron-system can be decomposed into 3 pair problems which have similar analytical solutions as the two- electron- system. The center of mass vector vanishes exactly in the classical ground state. Therefore, one should expect that our expansion works well for weak external fields or for systems, where after a Chern-Simons transformation and a proper mean field approximation (for finite systems!) the effective field is weak. Surprisingly, also for 3 particles in the small $\mathbf{R}$ limit, the Laughlin states, which belong to infinite fields, are among the analytically solvable solutions. 
The plan of this paper is the following. Sect.2 gives a survey on the results of the exact solutions of the electron pair problem. Because in this paper the three- electron- problem is traced back to three pair problems, this seems to be helpful. In Sect.3 we define an orthogonal transformation for the three- electron- problem which contains the center of mass $\mathbf{R}$ as a parameter, and then we expand the transformed Hamiltonian into a multipole series in $\mathbf{R}$. In Sect.4 it is shown that, in zero order in $\mathbf{R}$, the transformed Schrödinger equation can be solved exactly and analytically for a certain set of external fields and total angular momenta. In Sect.5, the eigenfunctions for 2 and 3 particles are written in an unified form and ad hoc generalized to arbitrary particle number. This expression is compared with the Laughlin and Jain states. In Sect.6 it is shown that the analytically solvable states are just those states where a cusp appears in the energy versus total angular momentum curve. The accuracy of the multi-pole expansion is tested in Sect.7 by calculation of the energy eigenvalues in first order perturbation theory in the dipole and the quadrupole term of the Hamiltonian. 


\section{Exact solutions for two electrons in an ho- mogeneous magnetic field}

In this section we summarize the results of a previous paper [13] on the twoelectron- problem and add some important subsequent unpublished findings. In particular, we add the asymptotic solutions to our former pattern, which had not been given the due attention in [13], and incorporate the electronelectron coupling constant $\beta$ explicitly, in order to be able to investigate the behavior of the exact solutions in varying the coupling strength. Completing and reviewing the two- electron- problem is important, because in the present work the three- electron- problem is traced back to three twoelectron- problems. It has been shown in [13] that the Schrödinger equation for two electrons in an homogeneous magnetic field plus an external parabolic scalar potential has exact analytical solutions for a certain infinite, but discrete set of field values (hereafter referred to as 'solvable fields') [t. Except for the asymptotic case of infinite external fields, which is part of this pattern, there is a one- to- one correspondence between exact solutions and solvable fields. Such solutions exist for singlet and triplet states as well as ground and excited states. A further qualitative feature is that these solutions occur, whenever a correlated state (with electron- electron interaction included) is degenerate with an uncorrelated one (without electron- electron interaction) [14. Moreover, for each total spin and orbital angular momentum quantum number as well as for a given degree of excitation (ground state, first excited state, etc.), there is an infinite series of solvable fields which converges to zero. This means that the solvable field values are dense at zero.

Now we are going to describe the general analytical form of the exact solutions. After introducing relative and center of mass coordinate

$$
\mathbf{r}=\mathbf{r}_{2}-\mathbf{r}_{1} \quad ; \quad \mathbf{R}=\frac{1}{2}\left(\mathbf{r}_{1}+\mathbf{r}_{2}\right)
$$

the Hamiltonian (in atomic units $\hbar=m=e=1$ )

$$
H=\sum_{i=1}^{2}\left\{\frac{1}{2}\left(\mathbf{p}_{i}+\frac{1}{c} \mathbf{A}\left(\mathbf{r}_{i}\right)\right)^{2}+\frac{1}{2} \omega_{o}^{2} r_{i}^{2}\right\}+\frac{\beta}{\left|\mathbf{r}_{2}-\mathbf{r}_{1}\right|}+H_{\text {spin }}
$$

\footnotetext{
${ }^{1}$ If we speak of 'fields' without specification to a special one, we mean the effective oscillator frequency $\sqrt{\omega_{0}^{2}+\left(\frac{\omega_{c}}{2}\right)^{2}}$, which is the relevant parameter.
} 
decouples exactly.

$$
H=2 H_{r}+\frac{1}{2} H_{R}+H_{\text {spin }}
$$

We follow the notation of 13 as long as not explicitly mentioned. $\omega_{0}$ is the oscillator frequency of the parabolic external confinement potential and $\mathbf{A}(\mathbf{r})=\frac{1}{2} \mathbf{B} \times \mathbf{r}$ the vector potential of the external magnetic field. The center of mass degree of freedom behaves like a quasi-particle in rescaled external fields and the quasi-particle of the relative coordinate is a particle in rescaled external fields plus a rescaled repulsive Coulomb field originating from the e- e- interaction. The Schrödinger equation of the first problem is trivial, the latter problem, which will be considered here, is described by the Hamiltonian (see eq.(5) in [13])

$$
H_{r}=\frac{1}{2}\left[\mathbf{p}+\frac{1}{c} \mathbf{A}_{r}\right]^{2}+\frac{1}{2} \omega_{r}^{2} r^{2}+\frac{\beta}{2 r}
$$

where $\omega_{r}=\frac{1}{2} \omega_{o}, \mathbf{A}_{r}=\frac{1}{2} \mathbf{A}(\mathbf{r})$. In polar coordinates $\mathbf{r}=(r, \alpha)$, the following ansatz for the eigenfunction

$$
\phi=\frac{e^{i m \alpha}}{\sqrt{2 \pi}} \frac{u(r)}{r^{1 / 2}} \quad ; \quad m=0, \pm 1, \pm 2, \ldots
$$

is justified, where the Pauli principle demands that $m$ is even or odd in the singlet and triplet state, respectively. The Schrödinger equation $H_{r} \phi(\mathbf{r})=$ $\epsilon_{r} \phi(\mathbf{r})$ gives rise to the radial Schrödinger equation for $u(r)$

$$
\left\{-\frac{1}{2} \frac{d^{2}}{d r^{2}}+\frac{1}{2}\left(m^{2}-\frac{1}{4}\right) \frac{1}{r^{2}}+\frac{1}{2} \tilde{\omega}_{r}^{2} r^{2}+\frac{\beta}{2 r}\right\} u(r)=\tilde{\epsilon}_{r} u(r)
$$

where $\tilde{\omega}_{r}=\frac{1}{2} \tilde{\omega}=\frac{1}{2} \sqrt{\omega_{o}^{2}+\left(\frac{\omega_{c}}{2}\right)^{2}}, \tilde{\epsilon}_{r}=\frac{1}{2} \tilde{\epsilon}=\epsilon_{r}-\frac{1}{4} m \omega_{c}, \omega_{c}=\frac{B}{c}$ and the solution is subject to the normalization condition $\int_{o}^{\infty} d r|u(r)|^{2}=1$. In dimensionless variables $r \rightarrow \sqrt{\tilde{\omega}_{r}} r$ and $\tilde{\epsilon}_{r} \rightarrow \tilde{\epsilon}_{r} / \tilde{\omega}_{r}$ the radial Schrödinger equation reads

$$
\begin{array}{r}
\left\{-\frac{1}{2} \frac{d^{2}}{d\left(\sqrt{\tilde{\omega}_{r}} r\right)^{2}}+\frac{1}{2}\left(m^{2}-\frac{1}{4}\right) \frac{1}{\left(\sqrt{\tilde{\omega}_{r}} r\right)^{2}}+\frac{1}{2}\left(\sqrt{\tilde{\omega}_{r}} r\right)^{2}+\frac{\beta}{\sqrt{\tilde{\omega}_{r}}} \frac{1}{2\left(\sqrt{\tilde{\omega}_{r}} r\right)}\right\} u(r)= \\
=\left(\frac{\tilde{\epsilon}_{r}}{\tilde{\omega}_{r}}\right) u(r)
\end{array}
$$

\footnotetext{
${ }^{2}$ The index ' $r$ ' and ' $R$ ' refers to the relative and c.m. coordinate systems, respectively
} 
The exactly solvable eigenfunctions have the following form

$$
u(r)=r^{|m|+\frac{1}{2}} p(r) e^{-\frac{1}{2} \tilde{\omega}_{r} r^{2}}
$$

where $p(r)$ is a finite polynomial

$$
p(r)=\sum_{\nu=0}^{(n-1)} a_{\nu} \cdot\left(\sqrt{\tilde{\omega}_{r}} r\right)^{\nu}
$$

with $n$ terms. The soluble fields and the corresponding eigenvalues are determined by the two requirements

$$
a_{n}=0 \quad ; \quad a_{n+1}=0
$$

which guarantee truncation of the power series and therefore normalizability of the eigenfunctions. It is clear from (17) that the two truncation conditions depend from the parameters only in the combination $\frac{\beta^{2}}{\tilde{\omega}_{r}}$ and $\frac{\tilde{\epsilon}_{r}}{\tilde{\omega}_{r}}$. Consequently, we have two equations (10) for effectively two parameters. Technically, we first calculate the solvable fields $\frac{\beta^{2}}{\tilde{\omega}_{r}}$ from (16) in [13] (with $\beta$ included) and then we get the corresponding eigenvalues from (17) in [13, which we rewrite here in the form

$$
\frac{\tilde{\epsilon}_{r}}{\tilde{\omega}_{r}}=|m|+n
$$

Note that (11) does not contain $\beta$ explicitly, but only through the fact that the solvable $\tilde{\omega}_{r}$ depend on beta. The calculation of the solvable fields is non-trivial (and not repeated here in detail), but when they are found, the calculation of the corresponding eigenvalues is simple through (11). Observe that the eigenvalues of (6) without e-e-interaction read [18]

$$
\frac{\tilde{\epsilon}_{r}}{\tilde{\omega}_{r}}=|m|+2 k+1
$$

where $k$ is the node number. Comparison of (11) and (12) shows the above mentioned degeneracies of the interacting system with the noninteracting one. With $n=2 k+1$, the interaction- free solutions fit into a generalized pattern of all analytical solutions. It can also be shown that equation (16) with (17) in [13], which defines the solvable $\frac{\beta^{2}}{\tilde{\omega}_{r}}$, has always the solution $\frac{\beta^{2}}{\tilde{\omega}_{r}}=0$ whenever $n$ is odd. As an example, see (20a) in [13 for the case 
$n=3$. (These infinite field solutions are not included in Tables 1 and 2 in [13.) It is also clear from physical reasoning that for infinite external fields the e-e-interaction has no influence on the eigensolutions, because the kinetic energy dominates. Therefore, solutions without e-e-interaction are exact for $\tilde{\omega}_{r} \rightarrow \infty$. Sometimes we shall call these solutions 'asymptotic' solutions.

The completed pattern has the following over- all- structure: For $n=1,2$ there is one solution (ground states), for $n=3,4$ we have two solutions (one ground and one excited state), etc. (see Figure 1). Generally, the soluble fields are the smaller the larger the corresponding $n$ is. For $n \rightarrow \infty$ the corresponding soluble $\tilde{\omega}_{r}$ converge to 0 .

Now we consider the eigenfunctions. The series $a_{\nu}$ is defined by a two step recursion relation which reads for the soluble states (insert (17) into (14) in [13])

$$
\begin{aligned}
a_{0} & =\text { normalization constant } \\
a_{1} & =\frac{1}{(2|m|+1)} \frac{\beta}{\sqrt{\tilde{\omega}_{r}}} a_{0} \\
a_{2} & =\frac{1}{4(|m|+1)}\left\{\frac{1}{(2|m|+1)} \frac{\beta^{2}}{\tilde{\omega}_{r}}-2(n-1)\right\} a_{0} \\
\ldots & \\
a_{\nu} & =\frac{1}{\nu(\nu+2|m|)}\left\{\frac{\beta}{\sqrt{\tilde{\omega}_{r}}} a_{\nu-1}+2(\nu-n-1) a_{\nu-2}\right\}
\end{aligned}
$$

It produces rather complicated expressions for larger $\nu$. The recursion can also be started at the other end with $a_{n-1}$ as a normalization constant.

The eigenfunctions of asymptotic solutions fit also into the generalized scheme. For $\frac{\beta^{2}}{\tilde{\omega}_{r}}=0$, the recursion relation (13) provides only non-vanishing coefficients with even index, meaning, that $p(r)$ is a function of $r^{2}$. If we insert $n=2 k+1$, and $\nu=2 p$ with $p=0,1,2, \ldots$ into (13) we obtain the recursion relation

$$
a_{p}=\frac{(p-k-1)}{p(p+|m|)} a_{p-1}
$$

which belongs to the Generalized Laguerre polynomials. 3

$$
p_{n=2 k+1, m}(r)=L_{k}^{|m|}\left(\tilde{\omega}_{r} r^{2}\right)
$$

\footnotetext{
${ }^{3}$ We use the definition in Abramowicz, Stegun; Handbook of Mathematical Functions
} 


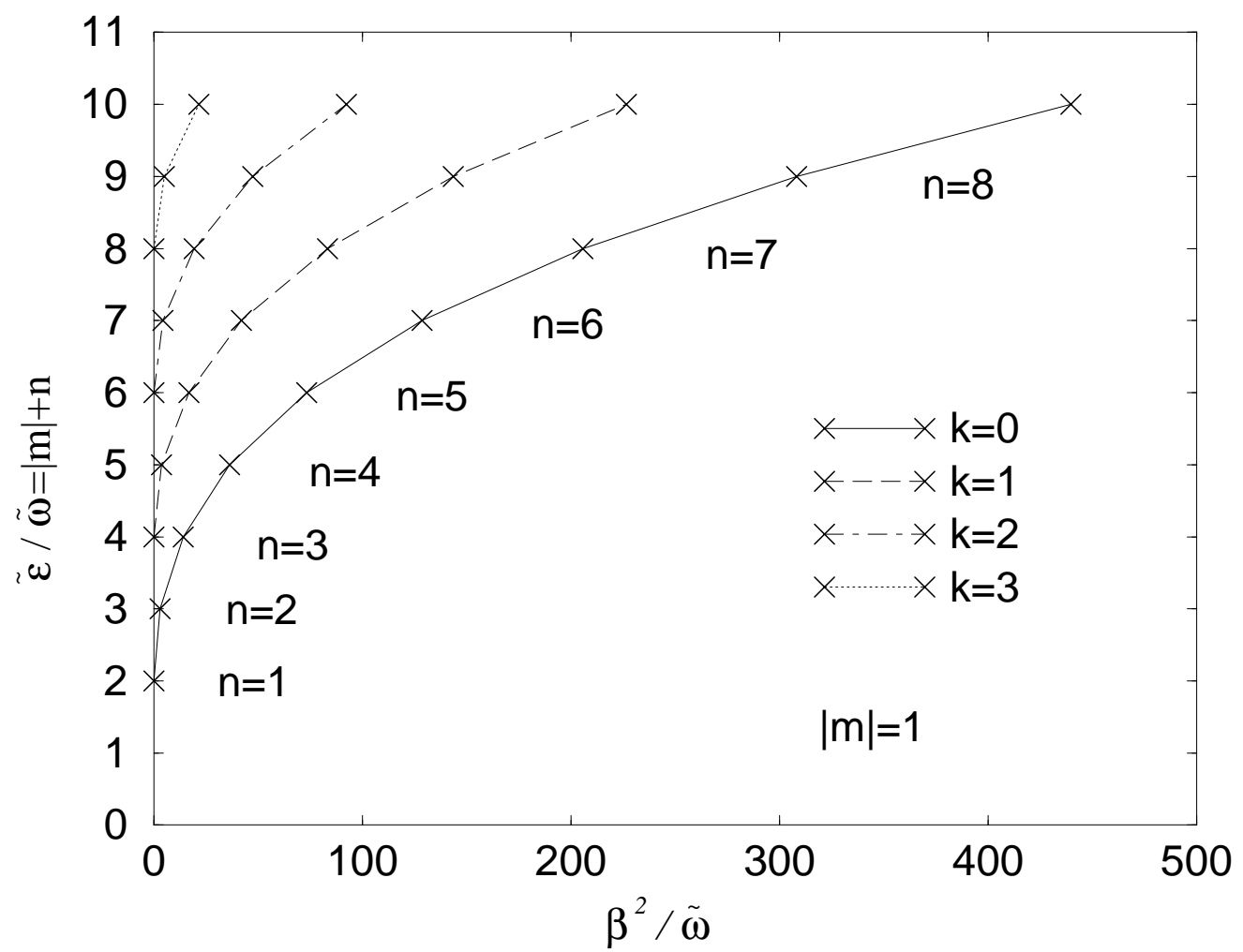

Figure 1: Reduced energies (energy over effective oscillator frequency) versus squared coupling constant over effective oscillator frequency for relative angular momentum $|m|=1$. The crosses indicate solvable states. The lines connect states with the same node number $k$. The termination index $n$ is the same for all horizontal rows of crosses with the same ordinate.

Consequently, the Generalized Laguerre polynomials are a special case of our polynomials $p_{n, m}(r)$. If we go in Fig.1 along the line for ground states $(k=0)$ from the left to the right, then the polynomials for the exactly solvable cases have the following form. For $n=1, p_{n=1, m}(r)=L_{0}^{|m|}\left(\tilde{\omega}_{r} r^{2}\right)$ is a constant, for $n=2$ (simplest case with finite fields) $p_{n=2, m}(r)$ is a linear function without a positive zero, for $n=3$ we have a quadratic function without positive zeros, etc. Analogously, the exact solutions for the first excited state are all 
polynomials with one node, but increasing order.

As an overview, we give the formulae for the simplest exact solutions of the completed pattern (see also (19) and (20) in [13] with $\beta$ included). For $n=1$ there is only the asymptotic solution

$$
\begin{aligned}
\frac{\beta^{2}}{\tilde{\omega}_{r}} & =0 \\
p(r) & =1
\end{aligned}
$$

For $n=2$ there is one finite- field solution

$$
\begin{aligned}
\frac{\beta^{2}}{\tilde{\omega}_{r}} & =2(2|m|+1) \\
p(r) & =\left[1+\frac{\beta r}{(2|m|+1)}\right]
\end{aligned}
$$

Both former solutions are ground states. For $n=3$ there is one asymptotic solution, which is a first excited state,

$$
\begin{aligned}
\frac{\beta^{2}}{\tilde{\omega}_{r}} & =0 \\
p(r) & =1-\frac{\tilde{\omega}_{r} r^{2}}{(|m|+1)}
\end{aligned}
$$

and one at finite fields, which is a ground state,

$$
\begin{aligned}
\frac{\beta^{2}}{\tilde{\omega}_{r}} & =4(4|m|+3) \\
p(r) & =\left[1+\frac{\beta r}{(2|m|+1)}+\frac{(\beta r)^{2}}{2(2|m|+1)(4|m|+3)}\right]
\end{aligned}
$$

The corresponding energies follow from (11), and we put $a_{0}=1$ for the constant term of the polynomial $p(r)$ without loss of generality.

In order to give an idea in what magnetic field range these exact solutions are located we consider the case without confinement $\left(\omega_{0}=0\right)$. Then $\tilde{\omega}_{r}=\omega_{c} / 4$, where all solvable frequencies given above are in effective atomic units a.u. ${ }^{*}\left(\hbar=m^{*}=\beta=1\right)$. On the other hand, for GaAs we have $B[$ Tesla $]=\omega_{c}\left[\right.$ a.u.* ${ }^{*} / 0.1363$. This means, that the largest finite solvable field ( for $n=2$ and $m=0$ ) is $\omega_{c}=2$ a.u. ${ }^{*}$ and $B=14.7$ Tesla. 
Now we add some words about the limit $\beta \rightarrow 0$. The wave functions of the asymptotic solutions $\left(\frac{\beta^{2}}{\tilde{\omega}_{r}}=0\right)$ do not depend on $\beta$ at all, indicating that they are robust against a variation of the $\mathrm{e}-\mathrm{e}-$ interaction. $\frac{\beta^{2}}{\tilde{\omega}_{r}}=0$ can be realized either by vanishing $\mathrm{e}^{-} \mathrm{e}-$ interaction $(\beta \rightarrow 0)$ or infinite fields $\left(\tilde{\omega}_{r} \rightarrow \infty\right)$. On the other hand, the wave functions of the finite- field- solutions $\left(\frac{\beta^{2}}{\tilde{\omega}_{r}}=\right.$ finite $)$ evolve steadily into non- interacting ones for $\beta \rightarrow 0$ indicating a strong dependence on the form of the $\mathrm{e}-\mathrm{e}-$ interaction.

If we insert for the center of mass system the ground state WF, then the total spatial WF in the particle coordinates reads (use (5), (8), and from [13 formula (8))

$$
\Phi\left(\mathbf{r}_{1}, \mathbf{r}_{2}\right)=\left(\mathbf{r}_{1}-\mathbf{r}_{2}\right)^{m} p_{n, m}\left(\left|\mathbf{r}_{1}-\mathbf{r}_{2}\right|\right) e^{-\frac{\tilde{\omega}}{2}\left(r_{1}^{2}+r_{2}^{2}\right)}
$$

where the first factor is a shorthand with the convention $\mathbf{r}^{m}=r^{|m|} e^{i m \alpha}$. In complex coordinates $z=x+i y, \bar{z}=x-i y$, and with the opposite sign for the angular momentum $\bar{m}=-m$ (compared with Laughlins notation [0] a 'bar' has been added), this means

$$
\begin{aligned}
& \mathbf{r}^{m}=z^{m} \quad \text { for } \quad m \geq 0 \\
& =\bar{z}^{\bar{m}} \quad \text { for } \quad m \leq 0
\end{aligned}
$$

Therefore, for $m \leq 0$, (24) reads in complex coordinates

$$
\Phi\left(\bar{z}_{1}, \bar{z}_{2}\right)=\left(\bar{z}_{1}-\bar{z}_{2}\right)^{\bar{m}} p_{n, \bar{m}}\left(\left|\bar{z}_{1}-\bar{z}_{2}\right|\right) e^{-\frac{\tilde{\omega}}{2}\left(\left|\bar{z}_{1}\right|^{2}+\left|\bar{z}_{2}\right|^{2}\right)}
$$

The solution for $n=1$ (infinite field, $p(x)=a_{0}=$ const.) agrees exactly with the Laughlin- WF, in particular, $\bar{m}=1$ is a determinant of two LLL functions, which corresponds to an uncorrelated full LLL.

For $m \geq 0$ we have

$$
\Phi\left(z_{1}, z_{2}\right)=\left(z_{1}-z_{2}\right)^{m} p_{n, m}\left(\left|z_{1}-z_{2}\right|\right) e^{-\frac{\tilde{\omega}}{2}\left(\left|z_{1}\right|^{2}+\left|z_{2}\right|^{2}\right)}
$$

which is the complex conjugate of (27) and therefore the solution for the opposite direction of the magnetic field, if $\omega_{0}=0$. In particular, $n=1$ and $m=1$ is a determinant of the two one- particle - states with $k=0$ and $m=0$

\footnotetext{
${ }^{4}$ Observe, our $m$ is the angular momentum quantum number itself and negative for the LLL.
} 
and $m=1$, in other words, it corresponds to the uncorrelated solution of two full LLs. In summary, the solutions for $n=1$ comprise the Laughlin WFs and the full LLs. It is tempting to assume that the other solutions $(n=2,3, \ldots)$ have some relation to the other Quantum Hall states, but until now there is no prove for it. (see also Sect. 5) 


\section{Approximate decoupling for three electrons}

The Hamiltonian for three electrons in an homogeneous magnetic field (vector potential $\mathbf{A}(\mathbf{r})$ ) and a harmonic scalar potential (oscillator frequency $\omega_{0}$ ) reads

$$
H=\sum_{i=1}^{3}\left[\frac{1}{2}\left(\frac{1}{i} \nabla_{i}+\frac{1}{c} \mathbf{A}\left(\mathbf{r}_{i}\right)\right)^{2}+\frac{1}{2} \omega_{o}^{2} r_{i}^{2}\right]+\sum_{i<k} \frac{\beta}{\left|\mathbf{r}_{i}-\mathbf{r}_{k}\right|}+H_{\text {spin }}
$$

where $H_{\text {spin }}=g \sum_{i=1}^{3} \mathbf{s}_{i} \cdot \mathbf{B}$. The goal of the following considerations is the decoupling of the Hamiltonian into a sum of independent Hamiltonians for quasi- particles.

For two electrons this happens automatically by introducing the center of mass and relative coordinate. This is mainly due to the peculiarity that there is only one interaction term $\frac{\beta}{\left|\mathbf{r}_{2}-\mathbf{r}_{1}\right|}$ which contains only one of the new coordinates $\mathbf{r}$ and $\mathbf{R}$. The peculiarity for three electrons, which can be taken advantage of, is the fact that the number of interaction terms is equal to the number of particles. This does not allow for an exact decoupling, but an approximate one into three noninteracting pairs plus a coupling term which is small in the strong correlation limit.

It is easily shown that an orthogonal transformation 9 leaves the kinetic energy in a homogeneous magnetic field and the potential energy in an external harmonic scalar potential, invariant. On the other hand, the center of mass (c.m.) of a classical system in the ground state vanishes and it is natural to assume that the c.m. in the high correlation limit can be treated as a small expansion parameter. Therefore, we look for an orthogonal transformation which transforms the $\mathrm{e}-\mathrm{e}-$ interaction in such a form that each term depends only on one of the new coordinates and the center of mass. Additionally, we demand that the center of mass is invariant under the transformation, which guarantees that if it is small in the original coordinates, so it is in the transformed ones. The transformation from the original coordinates $\mathbf{r}_{i}$ to the new

\footnotetext{
${ }^{5}$ Exactly speaking, for keeping the one- particle- terms of the Hamiltonian decoupled it suffices that the row vectors of the matrix in (30) are mutually orthogonal. However, using this more general type of transformation does not provide any advantage in our case, because the additional freedom destroys the symmetry among the particles.
} 
ones $\mathbf{x}_{i}$, which fulfills all these requirements, reads

$$
\left[\begin{array}{l}
\mathbf{x}_{1} \\
\mathbf{x}_{2} \\
\mathbf{x}_{3}
\end{array}\right]=\left[\begin{array}{ccc}
1 / 3 & a & b \\
b & 1 / 3 & a \\
a & b & 1 / 3
\end{array}\right]\left[\begin{array}{l}
\mathbf{r}_{1} \\
\mathbf{r}_{2} \\
\mathbf{r}_{3}
\end{array}\right]
$$

where $a=1 / 3-1 / \sqrt{3}$ and $b=1 / 3+1 / \sqrt{3}$ and its inverse is

$$
\left[\begin{array}{l}
\mathbf{r}_{1} \\
\mathbf{r}_{2} \\
\mathbf{r}_{3}
\end{array}\right]=\left[\begin{array}{ccc}
1 / 3 & b & a \\
a & 1 / 3 & b \\
b & a & 1 / 3
\end{array}\right]\left[\begin{array}{l}
\mathbf{x}_{1} \\
\mathbf{x}_{2} \\
\mathbf{x}_{3}
\end{array}\right]
$$

From (31) it follows that

$$
\begin{aligned}
& \mathbf{r}_{1}-\mathbf{r}_{2}=\sqrt{3}\left(\mathbf{X}-\mathbf{x}_{3}\right) \\
& \mathbf{r}_{2}-\mathbf{r}_{3}=\sqrt{3}\left(\mathbf{X}-\mathbf{x}_{1}\right) \\
& \mathbf{r}_{3}-\mathbf{r}_{1}=\sqrt{3}\left(\mathbf{X}-\mathbf{x}_{2}\right)
\end{aligned}
$$

so that the Hamiltonian in the new coordinates is

$$
H=\sum_{i=1}^{3}\left[\frac{1}{2}\left(\frac{1}{i} \nabla_{i}+\frac{1}{c} \mathbf{A}\left(\mathbf{x}_{i}\right)\right)^{2}+\frac{1}{2} \omega_{o}^{2} x_{i}^{2}+\frac{1}{\sqrt{3}} \frac{\beta}{\left|\mathbf{x}_{i}-\mathbf{X}\right|}\right]+H_{\text {spin }}
$$

where $\mathbf{X} \equiv \frac{1}{3} \sum_{i=1}^{3} \mathbf{x}_{i}=\mathbf{R}$ is the center of mass in the new coordinates. It is possible (but complicated) to take care of the Pauli principle in the new coordinates. It is much easier first to transform the wave functions (WF) back to the original coordinates and then do the antisymmetrization .

While being still exact, (33) is not exactly decoupled because $\mathbf{X}$ contains all coordinates. For $\mathbf{X}$ small compared with $\mathbf{x}_{i}$, the e-e-interaction term can be expanded in a multi-pole series

$$
V_{e e}=\sum_{l=0}^{\infty} V_{e e}^{(l)}
$$

where

$$
V_{e e}^{(0)}=\frac{\beta}{\sqrt{3}} \sum_{i=1}^{3} \frac{1}{\left|\mathbf{x}_{i}\right|}
$$




$$
\begin{aligned}
V_{e e}^{(1)} & =\frac{\beta}{\sqrt{3}} \sum_{i=1}^{3} \frac{\mathbf{X} \cdot \mathbf{x}_{i}}{\left|\mathbf{x}_{i}\right|^{3}} \\
V_{e e}^{(2)} & =\frac{\beta}{\sqrt{3}} \frac{1}{2} \sum_{i=1}^{3}\left[3 \frac{\left(\mathbf{X} \cdot \mathbf{x}_{i}\right)^{2}}{\left|\mathbf{x}_{i}\right|^{5}}-\frac{(\mathbf{X})^{2}}{\left|\mathbf{x}_{i}\right|^{3}}\right] \\
\ldots &
\end{aligned}
$$

In zero order in $\mathbf{X}$, the Hamiltonian $H^{(0)}$ is decoupled and can be solved exactly and in many cases even analytically (see below). The general strategy for amending the zero order result is to consider the multi-pole terms in perturbation theory. As a response to a frequently asked questions, we want to emphasize the following. The approximation $\mathbf{X}=0$ does not mean that the new coordinates $\mathbf{x}_{i}$ are no more independent. The one- particle part of the Hamiltonian is independent of $\mathbf{X}$ and still exact. $\mathbf{X}=0$ only means making an approximation to the $\mathrm{e}-\mathrm{e}-$ interaction term in $H$.

It might be interesting to note that transformation (30), if applied to an Hamiltonian with an external Coulombic potential $\frac{Z}{r_{i}}$ (instead of the oscillator potential $\frac{1}{2} \omega_{o}^{2} r_{i}^{2}$ ), transforms in zero order in $\mathbf{X}$ the e-e-interaction term and the potential energy in the external Coulombic potential into each other, i.e. leaves the Hamiltonian in lowest order virtually invariant. 


\section{Exact solution in zero order in the center of mass coordinate}

\subsection{Pair Equation}

After expanding the kinetic energy, the Hamiltonian in zero order in $\mathbf{X}$ reads

$$
H^{(0)}=\sum_{i=1}^{3} h_{i}+H_{\text {spin }}
$$

with an effective pair Hamiltonian

$$
h_{i}=-\frac{1}{2} \nabla_{i}^{2}+\frac{1}{2} \tilde{\omega}^{2} x_{i}^{2}+\frac{1}{2} \omega_{c} l_{i}+\frac{1}{\sqrt{3}} \frac{\beta}{\left|\mathbf{x}_{i}\right|}
$$

where $\tilde{\omega}=\sqrt{\omega_{0}^{2}+\left(\frac{1}{2} \omega_{c}\right)^{2}}, \omega_{c}=\frac{B}{c}$ is the cyclotron frequency, and $l_{i}$ is the orbital angular momentum operator. This gives rise to the definition of an effective pair equation

$$
h_{i} \phi_{q_{i}}\left(\mathbf{x}_{i}\right)=\epsilon_{q_{i}} \phi_{q_{i}}\left(\mathbf{x}_{i}\right)
$$

with the normalization condition

$$
\int d^{2} \mathbf{x}_{i}\left|\phi_{q_{i}}\left(\mathbf{x}_{i}\right)\right|^{2}=1
$$

The subscript $q_{i}$ at eigenvalues and eigenfunctions comprises all quantum numbers for the $i^{\text {th }}$ pair. In polar coordinates $(x, \alpha)$, the pair equation (40) is satisfied by the ansatz

$$
\phi=\frac{e^{i m \alpha}}{\sqrt{2 \pi}} \frac{u(x)}{x^{1 / 2}} \quad ; \quad m=0, \pm 1, \pm 2, \ldots
$$

$u(x)$ must satisfy the radial pair equation

$$
\left\{-\frac{1}{2} \frac{d^{2}}{d x^{2}}+\frac{1}{2}\left(m^{2}-\frac{1}{4}\right) \frac{1}{x^{2}}+\frac{1}{2} \tilde{\omega}^{2} x^{2}+\frac{\beta}{\sqrt{3} x}\right\} u(x)=\tilde{\epsilon} u(x)
$$

where $\tilde{\epsilon}=\epsilon-\frac{1}{2} m \omega_{c}$ and the solution is subject to the normalization condition $\int_{o}^{\infty} d x|u(x)|^{2}=1$. In analogy to the two- electron- problem, we now use for the radial part of the pair functions (42) the ansatz

$$
u(x)=x^{|m|+\frac{1}{2}} p(x) e^{-\frac{1}{2} \tilde{\omega} x^{2}}
$$


where $p(x)$ is a polynomial, which is finite for solvable states. In this way we obtain for the pair function (42) in polar coordinates $(x, \alpha)$

$$
\phi(\mathbf{x})=\frac{\mathbf{x}^{m}}{\sqrt{2 \pi}} p(x) e^{-\frac{1}{2} \tilde{\omega} x^{2}}
$$

where $\mathbf{x}^{m}$ is a shorthand for $x^{|m|} e^{i m \alpha}$ (see also Sect.2).

Because of the decoupling in zero order, the total eigenvalues and eigenfunctions of $H^{(0)}$ can be obtained from

$$
\begin{aligned}
& E_{q_{1}, q_{2}, q_{3}}=\epsilon_{q_{1}}+\epsilon_{q_{2}}+\epsilon_{q_{3}}+E_{\text {spin }} \\
& \Phi_{q_{1}, q_{2}, q_{3}}\left(\mathbf{x}_{1}, \mathbf{x}_{2}, \mathbf{x}_{3}\right)=\phi_{q_{1}}\left(\mathbf{x}_{1}\right) \cdot \phi_{q_{2}}\left(\mathbf{x}_{2}\right) \cdot \phi_{q_{3}}\left(\mathbf{x}_{3}\right)
\end{aligned}
$$

After inserting the transformation (32) into the spatial part of the total WF (47), we obtain in the original coordinates

$$
\begin{aligned}
\Phi_{q_{1}, q_{2}, q_{3}}= & \phi_{q_{1}}\left(\mathbf{R}-\frac{1}{\sqrt{3}}\left(\mathbf{r}_{2}-\mathbf{r}_{3}\right)\right) . \\
& \phi_{q_{2}}\left(\mathbf{R}-\frac{1}{\sqrt{3}}\left(\mathbf{r}_{3}-\mathbf{r}_{1}\right)\right) . \\
& \phi_{q_{3}}\left(\mathbf{R}-\frac{1}{\sqrt{3}}\left(\mathbf{r}_{1}-\mathbf{r}_{2}\right)\right)
\end{aligned}
$$

It is obvious that for any total WF the fields $\tilde{\omega}_{q_{i}}=\tilde{\omega}_{q}$ of the three pairs have to agree. If we insert (45) into (48), we end up with

$$
\begin{aligned}
\Phi_{q_{1}, q_{2}, q_{3}}= & \left(\mathbf{R}-\frac{1}{\sqrt{3}}\left(\mathbf{r}_{2}-\mathbf{r}_{3}\right)\right)^{m_{1}} p_{q_{1}}\left(\left|\mathbf{R}-\frac{1}{\sqrt{3}}\left(\mathbf{r}_{2}-\mathbf{r}_{3}\right)\right|\right) \\
& \left(\mathbf{R}-\frac{1}{\sqrt{3}}\left(\mathbf{r}_{3}-\mathbf{r}_{1}\right)\right)^{m_{2}} p_{q_{2}}\left(\mid \mathbf{R}-\frac{1}{\sqrt{3}}\left(\mathbf{r}_{3}-\mathbf{r}_{1}\right)\right) \\
& \left(\mathbf{R}-\frac{1}{\sqrt{3}}\left(\mathbf{r}_{1}-\mathbf{r}_{2}\right)\right)^{m_{3}} p_{q_{3}}\left(\left|\mathbf{R}-\frac{1}{\sqrt{3}}\left(\mathbf{r}_{1}-\mathbf{r}_{2}\right)\right|\right) \\
& \exp \left(-\frac{1}{2} \tilde{\omega}_{q} \sum_{i} \mathbf{r}_{i}^{2}\right)
\end{aligned}
$$

In order to simplify the exponential factor to the present form we used the special property of our orthogonal transformation $\sum_{i} \mathbf{x}_{i}^{2}=\sum_{i} \mathbf{r}_{i}^{2}$. This is still 
the most general result. It holds for ground and excited state and can be simplified in special cases. For analytically solvable finite-field solutions the quantum numbers of all three pairs have to agree, because the solvable fields $\tilde{\omega}_{q}$ for each pair depend on all quantum numbers contained in $q$. This implies in particular, that all angular momenta $m_{i}$ in (49) agree. For asymptotic solutions, the solvable $\tilde{\omega}$ does not depend on the angular momentum $m$ and the node number $k$ (or termination index $n=2 k+1$ ) of the corresponding pair. Therefore, we can construct analytical solutions from pair states with different quantum numbers, i.e. different $m_{i}$ and $k_{i}$. For asymptodic ground state solutions, which belong to $n_{i}=1$, (49) can be simplified by omitting the polynomials, because they are constants.

It should be mentioned that the eigenfunctions (47) of $H^{(0)}$ form a complete set and therefore they can be used as a basis for a numerical solution of the full Schrödinger equation. Their advantage compared with basis functions constructed from one- particle states [1] [17] [4] [4] is that they contain an $\mathrm{e}-\mathrm{e}-$ correlation cusp. As well known in Quantum Chemistry, the lack of this cusp in the basis functions gives rise to poor convergence in CI expansions. In the present paper, however, we consider perturbation theory for improving the zero order result beyond the strong correlation limit $\mathbf{R}=0$.

\subsection{Pauli Principle}

The WF (49) does not yet fulfill the antisymmetry requirement. Taking care of this is particularly important for finding the exclusion principles for our type of WF. The question is: for what combination of quantum numbers and parameter values does the antisymmetrized WF vanish by permutation symmetry? In order to keep formula length under control, we introduce some shorthands and conventions. Firstly, the exponential factor in (49) is fully symmetric with respect to permutations and therefore it can be simply omitted in the antisymmetrization procedure. Further, for the polynomial prefactor in (45) we introduce a single symbol

$$
t(\mathbf{x})=\mathbf{x}^{m} p(x)
$$

Then the spatial total WF can be written as

$$
\Phi_{q_{1}, q_{2}, q_{3}}\left(\mathbf{r}_{1}, \mathbf{r}_{2}, \mathbf{r}_{3}\right)=T_{q_{1}, q_{2}, q_{3}}\left(\mathbf{r}_{1}, \mathbf{r}_{2}, \mathbf{r}_{3}\right) \cdot \exp \left(-\frac{1}{2} \tilde{\omega}_{q} \sum_{i} \mathbf{r}_{i}^{2}\right)
$$




$$
\begin{aligned}
T_{q_{1}, q_{2}, q_{3}}\left(\mathbf{r}_{1}, \mathbf{r}_{2}, \mathbf{r}_{3}\right)= & t_{q_{1}}\left(\mathbf{R}-\frac{1}{\sqrt{3}}\left(\mathbf{r}_{2}-\mathbf{r}_{3}\right)\right) \\
& t_{q_{2}}\left(\mathbf{R}-\frac{1}{\sqrt{3}}\left(\mathbf{r}_{3}-\mathbf{r}_{1}\right)\right) . \\
& t_{q_{3}}\left(\mathbf{R}-\frac{1}{\sqrt{3}}\left(\mathbf{r}_{1}-\mathbf{r}_{2}\right)\right)
\end{aligned}
$$

Now, only the polynomial prefactor $T\left(\mathbf{r}_{1}, \mathbf{r}_{2}, \mathbf{r}_{3}\right)$ has to be antisymmetrized.

The implementation of the antisymmetrization procedure and the antisymmetrized WFs can be found in the Appendix. The most important qualitative result is that a simple exclusion principle exists only for $\mathbf{R}=\mathbf{0}$. It states that the WF vanishes by permutation symmetry:

in quartet states $S=\frac{3}{2}$ :

if at least two pair functions agree and if the total orbital angular momentum $M_{L}$ is even.

in doublet states $S=\frac{1}{2}$ :

if all three pair functions agree.

Because for analytical finite- field-solutions all quantum numbers of the 3 pairs have to agree, it follows from the Pauli principle (see above), that only quartet states (with parallel spins) can be given analytically. If $m$ is the angular momentum of the pair functions in (49) then the total orbital angular momentum in the solvable states is $M_{L}=3 \mathrm{~m}$. In zero order on $\mathbf{R}$, Pauli principle demands that $m=$ odd. For finite $\mathbf{R}$ there is no restriction from the Pauli principle and $m=$ integer. As will be shown in Section 6, it turns out that these solvable states are states where kinks in the curve $E$ versus $M_{L}$ occur and which bear the so called magic angular momenta (see Fig. 2). In short, only particular states can be solved analytically, but these states are the most interesting ones.

For asymptodic solutions (with $\beta^{2} / \tilde{\omega}=0$ ) all quantum numbers can be different, although the pair functions belong to the same external fields. This means that we can form total WFs from different pair functions and Pauli principle applies as given above. 


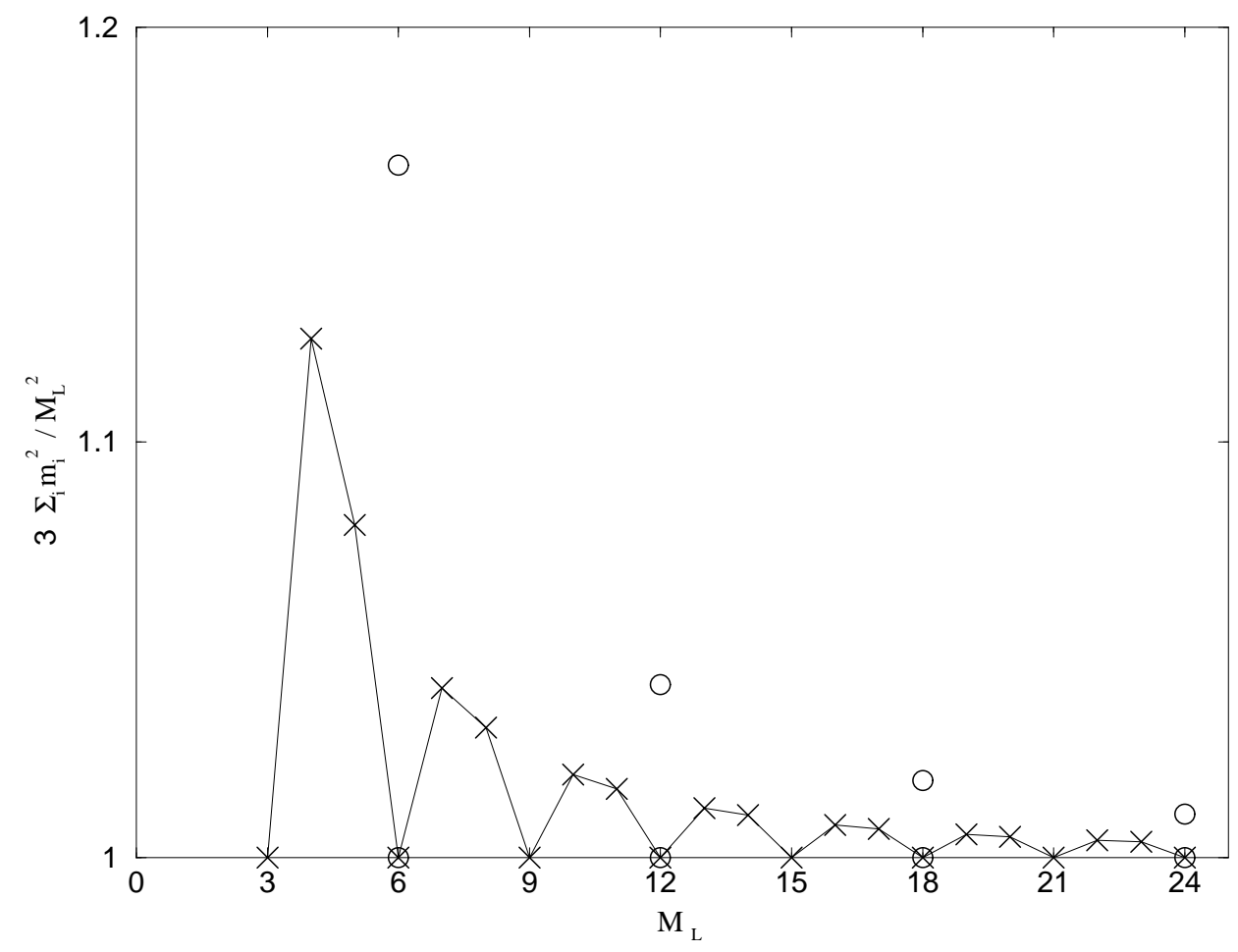

Figure 2: The second term of the total energy in second order Taylor approximation (76) divided by $c_{2} M_{L}^{2} / 3$ as a function of $M_{L}$ for the ground states (crosses). Crossed circles denote states which are forbidden by Pauli principle for $\mathbf{R}=0$ (Laughlin approximation), but allowed for finite $\mathbf{R}$. The states denoted by open circles are the ground states for $\mathbf{R}=0$ if the states underneath are forbidden. The region $M_{L}<3$ is omitted because it lies partly outside the scale and because it needs special consideration.

\subsection{Exact analytical solutions of the pair equation}

If we introduce in the radial equation for the relative coordinate in the twoelectron- case (6) the same parameters as used for 3 particles (i.e. $\tilde{\omega}$ and $\tilde{\epsilon}$ ), 
we obtain

$$
\left\{-\frac{1}{2} \frac{d^{2}}{d r^{2}}+\frac{1}{2}\left(m^{2}-\frac{1}{4}\right) \frac{1}{r^{2}}+\frac{1}{2}\left(\frac{\tilde{\omega}^{(N=2)}}{2}\right)^{2} r^{2}+\frac{\beta}{2 r}\right\} u^{(N=2)}(x)=\left(\frac{\tilde{\epsilon}^{(N=2)}}{2}\right) u^{(N=2)}(x)(52)
$$

For avoiding confusion, the parameters and the WF in the two- particleproblem have been given the extra superscript $N=2$. Comparison with the radial pair equation (43) shows us that we obtain solutions of (43) from solutions of (52) by simple rescaling.

The 'spectrum' of soluble $\tilde{\omega}$ follows from

$$
\tilde{\omega}=\frac{2}{3} \tilde{\omega}^{(N=2)}
$$

and the corresponding eigenvalues and eigenfunctions from

$$
\begin{aligned}
& \tilde{\epsilon}=\frac{2}{3} \tilde{\epsilon}^{(N=2)} \\
& u(x)=\sqrt{\frac{2}{\sqrt{3}}} u^{(N=2)}\left(r=\frac{2}{\sqrt{3}} x\right)
\end{aligned}
$$

The prefactor in (55) has been chosen to retain normalization of the radial pair function, if $u^{(N=2)}(r)$ is normalized. In any solution, the quotient $\frac{\tilde{\epsilon}}{\tilde{\omega}}$ is equal in both problems and given by (see [13])

$$
\frac{\tilde{\epsilon}}{\tilde{\omega}}=\frac{\tilde{\epsilon}^{(N=2)}}{\tilde{\omega}^{(N=2)}}=|m|+n
$$

where $m$ is the angular momentum and $(n-1)$ the highest power in the polynomial $p(x)$.

In this way we obtain from $(16-23)$ and $(53-55)$ the simplest exact solutions of (43) as follows:

For $n=1$ there is only the asymptotic solution

$$
\begin{aligned}
\frac{\beta^{2}}{\tilde{\omega}} & =0 \\
p(x) & =1
\end{aligned}
$$

For $n=2$ there is one finite- field- solution:

$$
\begin{aligned}
\frac{\beta^{2}}{\tilde{\omega}} & =\frac{3}{2}(2|m|+1) \\
p(x) & =1+\frac{\frac{2}{\sqrt{3}} \beta x}{(2|m|+1)}
\end{aligned}
$$


Both former solutions are ground states. For $n=3$ there is one asymptotic solution, which is a first excited state,

$$
\begin{aligned}
\frac{\beta^{2}}{\tilde{\omega}} & =0 \\
p(x) & =1-\frac{\tilde{\omega} x^{2}}{(|m|+1)}
\end{aligned}
$$

and one at finite fields, which is a ground state,

$$
\begin{aligned}
\frac{\beta^{2}}{\tilde{\omega}} & =3(4|m|+3) \\
p(x) & =1+\frac{\frac{2}{\sqrt{3}} \beta x}{(2|m|+1)}+\frac{\left(\frac{2}{\sqrt{3}} \beta x\right)^{2}}{2(2|m|+1)(4|m|+3)}
\end{aligned}
$$

The corresponding energies follow from (56), and we put the normalization constant $a_{0}=1$ without loss of generality. The pattern of solvable states agrees qualitatively with Fig.1. Only the abscissa- values of solvable states are shifted. Those, who are not yet convinced in the correctness of these solutions are recommended to check them by insertion into (43). 


\section{Comparison of our analytical solutions with Quantum Hall States}

In this section we confine ourselves to ground states and consider two cases separately. This will provide two different generalizations of the Laughlin WFs. We want to emphasize, however, that it would be possible (but not convenient) to include both cases in one formula. Secondly, we start with considering the case $\mathbf{R}=\mathbf{0}$ (zero order result) and add some words on the general case afterwards. Generally, the generalized formulae in this section comprise our analytical results for 2 particles and 3 particles. However, they could ad hoc be applied to any particle number and considered as trial functions.

The first case comprises all solutions for three electrons with equal pairangular- momenta in (49) and the two- electron result (24). In other words, it includes all finite- field solutions and and the asymptodic solutions with equal pair- angular- momenta. It can be written in the following unified form

$$
\Phi=\prod_{i<k}\left(\mathbf{r}_{i}-\mathbf{r}_{k}\right)^{m} p_{n, m}\left(\left|\mathbf{r}_{i}-\mathbf{r}_{k}\right|\right) \exp \left(-\frac{1}{2} \tilde{\omega}_{n, m} \sum_{l} \mathbf{r}_{l}^{2}\right)
$$

It should be remembered that, apart from the case $n=1$, the soluble field values $\tilde{\omega}_{n, m}$ and the polynomials $p_{n, m}(x)$ depend on the particle number.

Using complex coordinates as defined in Section 2, (65) can be reformulated as

$$
\begin{aligned}
\Phi & =\prod_{i<k}\left(\bar{z}_{i}-\bar{z}_{k}\right)^{\tilde{m}} p_{n, m}\left(\left|\bar{z}_{i}-\bar{z}_{k}\right|\right) \chi_{1}\left(\tilde{\omega}_{n, m}\right) \text { for } m \leq 0 \\
& =\text { complex conj. for } \quad m \geq 0
\end{aligned}
$$

where $\chi_{1}\left(\tilde{\omega}_{n, m}\right)$ is a Slater determinant of LLL states for the effective frequency $\tilde{\omega}_{n, m}$ and $\tilde{m}=|m|-1$. For $m \leq 0$ our WF can also be rewritten using the Laughlin WF [7]

$$
\Phi=\prod_{i<k} p_{n, m}\left(\left|\bar{z}_{i}-\bar{z}_{k}\right|\right) \Phi_{\nu=\frac{1}{\bar{m}}}^{\text {Laughlin }}\left(\tilde{\omega}_{n, m}\right)
$$

where the fields $\tilde{\omega}_{n, m}$ occur in the exponential factors of the Laughlin function instead of the infinite field used in the original Laughlin function. 
Our solutions (65) and the equivalent forms have the following properties: $\Phi$ fulfills the Pauli principle, if $m=o d d$ (and $\tilde{m}=$ even). It is an eigenfunction of the total angular momentum operator with eigenvalue $M_{L}=m \frac{N(N-1)}{2}$, where $N$ is the electron number. Apart from the asymptodic case $n=1$, it has components in higher LLs due to the $p(x)$-factors. In the case $n=1$ (where $p(x)=$ const and $\frac{1}{\tilde{\omega}_{1}}=0$ ) and $m \leq 0$, our WFs agree with the Laughlin states.

$$
\Phi_{\nu=\frac{1}{\bar{m}}}^{\text {Laughlin }}\left(\tilde{\omega}_{1}\right)=\prod_{i<k}\left(\bar{z}_{i}-\bar{z}_{k}\right)^{\bar{m}} \exp \left(-\frac{1}{2} \tilde{\omega}_{1} \sum_{l}\left|\bar{z}_{l}\right|^{2}\right)=\prod_{i<k}\left(\bar{z}_{i}-\bar{z}_{k}\right)^{\tilde{m}} \chi_{1}\left(\tilde{\omega}_{1}\right)(68)
$$

For comparison, we also quote the Jain ansatz [8]

$$
\Phi_{\nu}^{J a i n}=\mathcal{P}_{L L L} \prod_{i<k}\left(\bar{z}_{i}-\bar{z}_{k}\right)^{\tilde{m}} \chi_{\nu^{*}}\left(\tilde{\omega}_{1}\right)
$$

where $\frac{1}{\nu}= \pm \frac{1}{\nu^{*}}+\tilde{m}, \mathcal{P}_{L L L}$ is a projection operator onto the LLL and the determinant $\chi_{\nu^{*}}\left(\tilde{\omega}_{1}\right)$ is for $\nu^{*}$ full LLs and taken at the asymptotically infinite frequency $\tilde{\omega}_{1}$. Apart from the special cases discussed above, (66) and (69) do not seem to be fully equivalent. At least, both treatments contain the Laughlin WF as a special case, and there is the vague similarity that both have to do with higher LL components.

The next property will be discussed using the formulae for $N=3$, but it would also apply to the corresponding generalized trial functions. If we go beyond the $\mathbf{R}=0$ approximation, i.e. if we calculate the form of the pair functions in the transformed space $\mathbf{x}_{i}$ in zero order in $\mathbf{X}=\mathbf{R}$, but use the full back - transformation to the original coordinates $\mathbf{r}_{i}$ involving a finite $\mathbf{R}$, then the solvable eigenfunctions are those in (49) with $m_{1}=m_{2}=m_{3}=m$. Firstly, we have to remind that this function has to be antisymmetrized as discussed in the Appendix, because it is not automatically antisymmetric for $m=$ odd as in the case $\mathbf{R}=0$. This provides a complicated expression. Due to this antisymmetrization it has simple zeros wherever two coordinates agree. However, there are no $m$ - fold zeros as in the Laughlin WF, because the factors $\left(\mathbf{R}-\frac{1}{\sqrt{3}}\left(\mathbf{r}_{i}-\mathbf{r}_{k}\right)\right)$ do not vanish if two coordinates agree. This holds for all solutions (finite and infinite field solutions). This is a feature which agrees qualitatively with the Jain functions. 
The second case differs from the first case only for $N>2$. For the asymptodic solutions in (49), different $m_{i}$ are allowed, and for the ground state the polynomials can be omitted because they are constants. After introducing a new indexing for the angular momenta, which is more appropriate for the $N$-particle system, we obtain from (49)

$$
\begin{aligned}
\Phi & =\prod_{i<k}\left(\mathbf{r}_{i}-\mathbf{r}_{k}\right)^{m_{i k}} \exp \left(-\frac{1}{2} \tilde{\omega}_{1} \sum_{l} \mathbf{r}_{l}^{2}\right) \\
& =\prod_{i<k}\left(\bar{z}_{i}-\bar{z}_{k}\right)^{\bar{m}_{i k}} \exp \left(-\frac{1}{2} \tilde{\omega}_{1} \sum_{l}\left|\bar{z}_{l}\right|^{2}\right)
\end{aligned}
$$

The second equation holds for $m_{i k} \leq 0$. For general $m_{i k}$, this function has to be antisymmetrized. For 3 particles, the antisymmetrized result is discussed in Section 4.2 and given in the Appendix. In this special case Pauli principle demands that $M_{L}=o d d . \Phi$ in (70) is an eigenfunction of the total orbital angular momentum with eigenvalue $M_{L}=\sum_{i<k} m_{i k}$, and lies completely within the LLL. It is apparent that the special case of equal $m_{i k}=m=o d d$ agrees with the Laughlin states. The changes produced by the extension of the considerations to finite $\mathbf{R}$ are analogous to the first case.

A more detailed investigation of the applicability of the two generalizations (65) and (70) to $N$-particle systems and in particular Quantum Hall states will be the aim of a separate work. 


\section{Approximate analytical solution of the pair equation and magic angular momenta for three electrons}

In order to avoid misunderstandings, it should be told at the very beginning, why we are looking for approximate solutions, if there are exact ones. The answer is that the exact solutions exist only for certain fields and states, whereas the solutions of this subsection are for all parameters and all states. Although the exactly soluble states are the most interesting ones, the rest is necessary to prove certain cusp properties of the exactly solvable ones. From here on we consider only the case $\beta=1$ and finite fields.

As shown in [13], sect.3.2, the effective potential in the radial pair equation (43) can be expanded around its minimum into a Taylor series to second order. This cannot be accomplished fully analytically because the minimum position results from the zeros of a forth order polynomial equation. It is possible, however, to establish the approximate effective potential to order

$r_{0}^{-1}$, where $r_{0}^{-1}=\left(\frac{9}{8} \tilde{\omega}\right)^{1 / 3}$ is a small parameter in the strong correlation limit. The eigenvalues of the resulting oscillator equation can then be given analytically with the result

$$
\epsilon=c_{0}+c_{1} m+c_{2} m^{2}+O\left(r_{0}^{-2}\right)
$$

where

$$
\begin{aligned}
& c_{0}=\left[1-\frac{1}{43^{1 / 3}} \tilde{\omega}^{2 / 3}\right]\left[\frac{1}{2}(3 \tilde{\omega})^{2 / 3}+\sqrt{3} \tilde{\omega}\left(k+\frac{1}{2}\right)\right] \\
& c_{1}=\frac{1}{2} \omega_{c} \\
& c_{2}=3^{-1 / 3} \tilde{\omega}^{2 / 3}\left[\frac{1}{2}(3 \tilde{\omega})^{2 / 3}+\sqrt{3} \tilde{\omega}\left(k+\frac{1}{2}\right)\right]
\end{aligned}
$$

where $k$ is the node number. This provides a total energy of

$$
E=3 c_{0}+c_{1} M_{L}+c_{2}\left(m_{1}^{2}+m_{2}^{2}+m_{3}^{2}\right)
$$

where $M_{L}=m_{1}+m_{2}+m_{3}$. It is clear that the Taylor expansion is the better the more symmetric the effective potential is. Therefore it gets poorer with increasing $m$ and increasing $\tilde{\omega}$. Nevertheless, this formula gives a qualitative understanding of the magic angular momenta. If we are interested in the 
ground state for a given $M_{L}$, it is clear from (76) that it is formed by that set of $m_{i}$ for which the sum of the squares of the $m_{i}$ is minimal for a given sum of the $m_{i}$. This demand is met if the $m_{i}$ are 'as equal as possible'. As a example, for $M_{L}=2$ there are two sets which provide the same $M_{L}$, namely (002) and (011), with the latter forming the ground state. It is also clear that the total momenta of the form $M_{L}=3 m$ (multiple of three) play a special role because all three $m_{i}$ can be equal in this case, but not for the other $M_{L}$. Fig. 1 shows the third (and only discontinuous) term of (76) as a function of $M_{L}$. It is obvious that this curve has kinks whenever it is possible that all three $m_{i}$ are equal, i.e. for $M_{L}=3 m$ with $m=0,1,2, \cdots$. On the other hand, we learned, that equal $m_{i}$ is a prerequisite for analytical solutions. Thus we conclude that the solvable states are always cusp states.

Observe, that in the limit $\mathbf{R}=0$ the Pauli principle demands that even $m$ are forbidden (see Sect. 4.2). We also want to remind (see Sect. 5) that we obtain the Laughlin WF in the $\mathbf{R}=0$ limit. If we go beyond this approximation, the total WFs (48) can be antisymmetrized for any values of the $m_{i}$. These facts elucidate the origin for the well known problem that exact diagonalization procedures provide cusps also for those $M_{L}$, which correspond to even denominator Laughlin states [1]. This shows that the Laughlin ansatz (if applied to finite systems) has an additional symmetry (produced by putting $\mathbf{R}=0$ ), which is not present in exact solutions. These conclusions are not in contradiction with the symmetry considerations in [15 and [16]. The latter papers find formula for the cusp (or magic) angular momenta, provided, the states under investigation are not forbidden by Pauli's principle. They do not have (and need) any explicit expression for the wave function. By the way, their general notion on the eigenstates is consistent with our small- $\mathbf{R}$ expansion. The harmonic approximation used in this section is also related to the harmonic approach used in [17], but not fully equivalent. However, our analytic approximations derived in Sect.s 3 and 4 is not harmonic. 


\section{First order perturbation theory and accu- racy of the strong correlation expansion}

Now we are going to calculate the contributions of the dipole and quadrupole term (36) and (37) of the e-e-interaction to the total energy in first order 9 perturbation theory, i.e.

$$
\Delta E^{(l)}=<\Phi\left|V_{e e}^{(l)}\right| \Phi>
$$

where the zero order wave function is generally given by (87). For quartet states, where the total WF is just a product of spatial and spin part and for calculating matrix elements, the total WF in (77) can be replaced by the unsymmetrized spatial part. (It is simpler, however, to do the integrations in the transformed $\mathbf{x}_{i}$-coordinates rather than in the $\left.\mathbf{r}_{i}\right)$. The further calculation is straight forward and provides

$$
\begin{aligned}
\Delta E^{(1)} & =\frac{1}{3 \sqrt{3}} \sum_{k=1}^{3} M_{m_{k}}^{(-1)} \\
\Delta E^{(2)} & =\frac{1}{9 \sqrt{3}}\left[\sum_{k=1}^{3} M_{m_{k}}^{(-1)}-\frac{1}{2} \sum_{k=1}^{3} \sum_{l(\neq k)=1}^{3} M_{m_{k}}^{(-3)} M_{m_{k}}^{(2)}\right]
\end{aligned}
$$

where we defined moments

$$
M_{m}^{(k)}=\int_{0}^{\infty} d x x^{k}\left[u_{m}(x)\right]^{2}
$$

with $u_{m}(x)$ being the radial part defined in (42) and given explicitly in special cases using (60) and (64). If all three angular momenta agree: $m_{1}=m_{2}=m_{3} \equiv m$, the result simplifies to

$$
\begin{aligned}
\Delta E^{(1)} & =\frac{1}{\sqrt{3}} M_{m}^{(-1)} \\
\Delta E^{(2)} & =\frac{1}{3 \sqrt{3}}\left[M_{m}^{(-1)}-M_{m}^{(-3)} M_{m}^{(2)}\right]
\end{aligned}
$$

It should be noted that the moment $M_{m}^{(-3)}$, appearing in the second order contribution, diverges for $m=0$. This is because for $x \rightarrow 0$ the radial pair

\footnotetext{
$\mathbf{R}$

${ }^{6} \mathrm{By}$ first order we mean first order in all multi-pole corrections, but not first order in
} 
function goes as $u_{m}(x) \rightarrow x^{\left(|m|+\frac{1}{2}\right)}$ and thus the integrand in (80) behaves like $x^{-2}$ for small $x$. That is why the results for $m=0$ are missing in Table 2 .

For a test of the accuracy of our small- $\mathbf{R}$ expansion we use analytically solvable states only, i.e. quartet states with $M_{L}=3 \mathrm{~m}$. We do this for magnetic field only (i.e. $\omega_{0}=0$ ), because the confinement can be included afterwards by a simple rescaling of the parameters. For $\omega_{0}=0$ it follows from (56) and the definitions of $\tilde{\omega}$ and $\tilde{\epsilon}$ that the zero order (in $\mathbf{R}$ ) energy per electron reads

$$
\frac{E^{(0)}}{3 \omega_{c}}=\frac{(m+|m|)}{2}+\frac{n}{2}
$$

and, for comparison, the trivial result without e-e-interaction is 18

$$
\frac{E^{(n o n-i n t)}}{3 \omega_{c}}=\frac{(m+|m|)}{2}+k+\frac{1}{2}
$$

The formulae for the corrections in first and second order $\Delta E^{(1)}$ and $\Delta E^{(2)}$, respectively, are given in (81) and (82). Table 1 and 2 show the results for fixed $m$ and varying $n$ and fixed $n$ and different $m$, respectively. $E^{(\text {Taylor })}$ is the result using the Taylor expansion of the effective potential in the radial Schrödinger equation as described in Sect. 6. Its agreement with the exact (analytical) solution of the radial Schrödinger equation $E^{(0)}$ gives an account of the accuracy of the Taylor expansion.

We conclude the following.

i) Comparison of $E^{(n o n-i n t)}$ and $E^{(0)}$ shows the contribution of the Coulomb interaction energy to the total energy. As to be expected, it grows with decreasing $\omega_{c}$ and is tremendous for small $\omega_{c}$ (e.g. ten times larger than the kinetic energy for $n=10$ i.e. $\omega_{c} \approx 10^{-3}$ ).

ii) For small $|m|$, the Taylor approximation provides a good tool for solving the radial Schrödinger equation. For large $|m|$ the effective potential becomes so unsymmetric with respect to its minimum that its result goes fatally wrong.

iii) The analytical zero order result $E^{(0)}$, which is the main achievement of this paper, compares pretty well with the most precise result $E^{(2)}$. This is mainly due to the cancellation of $\Delta E^{(1)}$ and $\Delta E^{(2)}$. The maximum error is of the order of $10 \%$. 
Table 1: Total energy per electron in units of $\omega_{c}$ for analytically solvable quartet states with total orbital angular momentum $M_{L}=-3(m=-1)$ for $\omega_{0}=0$ (magnetic field only). $E^{(n o n-i n t)}$ is the energy without electronelectron interaction, $E^{(\text {Taylor })}$ is the total energy where the effective potential in the radial pair equation is treated in second order Taylor expansion (76), and $E^{(0)}$ is the exact result in zero order in $\mathbf{R}$ as given in (83). $\Delta E^{(1,2)}$ are the dipole and quadrupole contributions given in (81) and (82), and $E^{(2)}$ is the sum of the three former contributions. The Zemann energy is omitted.

\begin{tabular}{|r|r|r|r|l|r|r|r|}
\hline $\mathrm{n}$ & $\omega_{c}$ & $\frac{E^{(\text {non-int })}}{3 \omega_{c}}$ & $\frac{E^{(\text {Taylor })}}{3 \omega_{c}}$ & $\frac{E^{(0)}}{3 \omega_{c}}$ & $\frac{\Delta E^{(1)}}{3 \omega_{c}}$ & $\frac{\Delta E^{(2)}}{3 \omega_{c}}$ & $\frac{E^{(2)}}{3 \omega_{c}}$ \\
\hline 2 & $\frac{4}{9}=444.444$ E-3 & 0.5 & 1.03796 & 1 & 0.154332 & -0.101713 & 1.052619 \\
3 & $\frac{2}{21}=95.2381$ E-3 & 0.5 & 1.49531 & 1.5 & 0.290373 & -0.126757 & 1.663616 \\
5 & 18.1896 E-3 & 0.5 & 2.49055 & 2.5 & & & \\
10 & 2.20940 E-3 & 0.5 & 4.99381 & 5 & & & \\
15 & 0.655360 E-3 & 0.5 & 7.49561 & 7.5 & & & \\
\hline
\end{tabular}

Table 2: Total energy per electron in units of $\omega_{c}$ for analytically solvable quartet states for $\omega_{0}=0$ (magnetic field only) as a function of $m$ with $M_{L}=$ $3 m$. (Only odd $m$ are compatible with the Pauli principle.) All solutions belong to $n=2$. The meaning of the other column heads is as in Table 1 .

\begin{tabular}{|r|r|c|c|r|r|l|r|}
\hline $\mathrm{m}$ & $\omega_{c}$ & $\frac{E^{(\text {non-int })}}{3 \omega_{c}}$ & $\frac{E^{(\text {Taylor })}}{3 \omega_{c}}$ & $\frac{E^{(0)}}{3 \omega_{c}}$ & $\frac{\Delta E^{(1)}}{3 \omega_{c}}$ & $\frac{\Delta E^{(2)}}{3 \omega_{c}}$ & $\frac{E^{(2)}}{3 \omega_{c}}$ \\
\hline 10 & $\frac{4}{63}=0.063$ & 10.5 & 21.4662 & 11 & 0.164717 & -0.00834761 & 11.15637 \\
$\ldots$ & & & & & & & \\
3 & $\frac{4}{21}=0.190$ & 3.5 & 5.06036 & 4 & 0.161018 & -0.0283146 & 4.132703 \\
2 & $\frac{4}{15}=0.266$ & 2.5 & 3.43552 & 3 & 0.158918 & -0.0435331 & 3.115385 \\
1 & $\frac{4}{9}=0.444$ & 1.5 & 2.03796 & 2 & 0.154332 & -0.101713 & 2.052619 \\
\hline 0 & $\frac{4}{3}=1.333$ & 0.5 & 0.892263 & 1 & 0.136400 & & \\
\hline-1 & $\frac{4}{9}=0.444$ & 0.5 & 1.03796 & 1 & 0.154332 & -0.101713 & 1.052619 \\
-2 & $\frac{4}{15}=0.266$ & 0.5 & 1.43552 & 1 & 0.158918 & -0.0435331 & 1.115385 \\
-3 & $\frac{4}{21}=0.190$ & 0.5 & 2.06036 & 1 & 0.161018 & -0.0283146 & 1.132703 \\
$\ldots$ & & & & & & & \\
-10 & $\frac{4}{63}=0.063$ & 0.5 & 11.4662 & 1 & 0.164717 & -0.00834761 & 1.156369 \\
\hline
\end{tabular}




\section{Summary and discussion}

We found that the two-dimensional Schrödinger equation for 3 electrons in an homogeneous magnetic field (perpendicular to the plane) and a parabolic scalar confinement potential has exact analytical solutions in the strong correlation limit, where the expectation value of the center of mass vector is small compared with the average distance between the electrons. These analytical solutions exist only for certain discrete values of the external fields $\tilde{\omega}$. For finite external fields, analytical solvability demands that all three pairangular- momenta agree what leads to total angular momenta $M_{L}=3 \mathrm{~m}$ with $m=$ integer. In zero order in $\mathbf{R}$, Pauli principle allows equal pairangular- momenta only for parallel spins and $m=o d d$. The analytically solvable states are always cusp states, i.e. states where $E\left(M_{L}\right)$ has a cusp.

Further, these special analytical solutions for 3 particles and the exact analytical solutions for 2 particles could be written in a unified form, which contains only products over coordinate combinations $\prod_{i<k}$ and sums $\sum_{i}$. Conveniently, instead of using one formula we consider two cases (65) and (70). These formulae, when ad hoc generalized to $\mathrm{N}$ coordinates, can be discussed as ansatzes for the wave function of the N-particle system. These ansatzes fulfill the following demands: they are exact for two particles and for 3 particles in the limit of small $\mathbf{R}$, and they are eigenfunction of the total orbital angular momentum. The Laughlin functions are special case, or in other words, both formulae provide two different generalizations of the Laughlin functions.

Until now we know mainly that our WFs are analytically solvable states (within the approximations discussed above). It is also clear that states for an infinitesimally varied $\tilde{\omega}$ look quite different, i.e. the finite polynomials have to be replaced by polynomials with an infinite number of terms. For $\mathrm{N}=2$ and 3 these 'neighboring' states are even explicitly known. It is not yet shown, however, if these special states have generally something to do with the Quantum Hall states (which show similar singular features), or if any physical quantity has any special feature in these soluble states. One encouraging fact is that the Laughlin states are special cases of our states. In prospect, it is possibly a good idea to look for similar exact analytical solutions in a spherical geometry instead of the disk geometry used here, because it is easier then to attribute a filling factor to each eigensolutions. If there is a connection between our states and Quantum Hall states, this would 
imply some kind of inherent super-symmetry in the Quantum Hall states. Now we want to compare our treatment of three electrons with Laughlins [7]. Both approaches are approximate. Whereas he forms WF by antisymmetrization of one- particle states of the LLL, which is expected to be good for strong fields, we established an expansion, which is good in the strong correlation limit and which contains in general higher LL components. Consequently, we obtained a richer variety of solutions comprising the Laughlin states as special cases. 


\section{Appendix: Pauli Principle}

In order the obtain familiar looking formula, we rename $T \rightarrow \Phi$ and $t \rightarrow \phi$. The question here is, how the properly symmetrized spatial part $\Phi$ has to be supplemented by an appropriate spin part in order to obtain a wave function which is eigenfunction of $\mathbf{S}^{2}$ and $S_{z}$ (with quantum numbers $S$ and $M_{S}$ ) and which satisfies the Pauli principle. $\mathbf{S}=\sum_{i=1}^{N} \mathbf{s}_{i}$ is the total spin operator for all particles. For more than two particles this is a well established, but nontrivial procedure. The source of the difficulty is the fact that the spin space can be degenerate, i.e. there is more than one orthogonal spin eigenfunction $X_{i},(i=1, \ldots f)$ for given $S$ and $M$ (see Table 3 for $\mathrm{N}=3$ ).

Table 3:

Standard spin eigenfunctions for $\mathrm{N}=3 . \quad \alpha$ and $\beta$ are the one-particle spin eigenfunctions for spin up and down, respectively. The first factor of a product of one-particle functions carries the spin variable '1', the second factor carries '2' etc.

\begin{tabular}{|l|l|l|l|l|}
\hline $\mathrm{S}$ & $M_{S}$ & $\mathrm{i}$ & $X_{i}$ & permutation sym. \\
\hline$\frac{1}{2}$ & $+\frac{1}{2}$ & 1 & $\frac{1}{\sqrt{6}}[-(\alpha \beta+\beta \alpha) \alpha+2 \alpha \alpha \beta]$ & symmetric for (12) \\
& $-\frac{1}{2}$ & & $\frac{1}{\sqrt{6}}[+(\beta \alpha+\alpha \beta) \beta-2 \beta \beta \alpha]$ & \\
\hline$\frac{1}{2}$ & $+\frac{1}{2}$ & 2 & $\frac{1}{\sqrt{2}}(\alpha \beta-\beta \alpha) \alpha$ & antisymmetric for (12) \\
& $-\frac{1}{2}$ & & $-\frac{1}{\sqrt{2}}(\beta \alpha-\alpha \beta) \beta$ & \\
\hline$\frac{3}{2}$ & $+\frac{3}{2}$ & 1 & $\alpha \alpha \alpha$ & symmetric for all $\mathrm{P}$ \\
& $+\frac{1}{2}$ & & $\frac{1}{\sqrt{3}}[\alpha(\alpha \beta+\beta \alpha)+\beta \alpha \alpha]$ & \\
& $-\frac{1}{2}$ & & $\frac{1}{\sqrt{3}}[\beta(\beta \alpha+\alpha \beta)+\alpha \beta \beta]$ & \\
& $-\frac{3}{2}$ & & $\beta \beta \beta$ & \\
\hline
\end{tabular}


Generally, the total WF $\Psi$, which fulfills our demands, can be calculated from (see e.g. [19])

$$
\Psi_{i}(1,2,3)=\mathcal{A}_{a} X_{i}(1,2,3) \Phi(1,2,3) \quad i=(1, \ldots, f)
$$

with the antisymmetrizer

$$
\mathcal{A}_{a}=\frac{1}{\sqrt{N !}} \sum_{\mathcal{P}}(-1)^{p} \mathcal{P}
$$

and $\mathcal{P}$ is a permutation operator. $f$ is the dimension of the degenerate spin space $\left(f=1\right.$ and 2 for $S=\frac{3}{2}$ and $\frac{1}{2}$, respectively, for $\left.\mathrm{N}=3\right)$ and $(-1)^{p}$ is the parity of the permutation $\mathcal{P}$. The quantum numbers $S$ and $M_{S}$ of the spin part $X_{i}$ as well as the quantum numbers of the spatial part $\Phi$ are not indicated. The arguments $(1,2,3)$ are spin or spatial coordinates depending on the function in question. Although being correct, (85) does not reveal the inherent permutation symmetry of the total WF. An equivalent symmetrized form is (see e.g. [19])

$$
\Psi_{i}=\frac{1}{\sqrt{f}} \sum_{k=1}^{f} X_{k} \cdot \Phi_{k i}^{s}
$$

where the symmetrized spatial function is defined as

$$
\Phi_{k i}^{s}=\sqrt{\frac{f}{N !}} \sum_{\mathcal{P}}(-1)^{p} U_{k i}(\mathcal{P}) \mathcal{P} \Phi
$$

and $U_{k i}(\mathcal{P})$ is an irreducible representation matrix of the permutation group for permutation $\mathcal{P}$ given in Table 4 . It is convenient to define column vectors $\boldsymbol{\Phi}_{i}^{s}$ (with $i=1,2$ ) of the matrix $\Phi_{k i}^{s}$. Then the $i^{t h}$ vector comprises all spatial information about the $i^{\text {th }}$ of the orthogonal states $\Psi_{i}$.

Quartet $S=\frac{3}{2}$

Because of $f=1$ and because the spin eigenfunctions are symmetric against all permutations, the symmetrized spatial function is totally antisymmetric and we have

$$
\Psi=X \Phi^{s} ; \quad \Phi^{s}=\mathcal{A}_{a} \Phi
$$

which is reminiscent of the permutation symmetry of the triplet state for $N=2$. If we insert the solution (51) for $\Phi$ we obtain a lengthy expression 
Table 4: Irreducible representation matrices $\mathbf{U}(\mathcal{P})$ for $\mathrm{N}=3$. For class and cyclic permutation symbols see [19].

\begin{tabular}{|c|c|c|c|}
\hline class & $\mathrm{P}$ & & $\mathbf{U}(\mathrm{P})$ \\
\hline$\left[1^{3}\right]$ & $\varepsilon$ & & $\begin{array}{ll}1 & 0 \\
0 & 1\end{array}$ \\
\hline \multirow[t]{3}{*}[2,1]{} & $(1,2)$ & & $\begin{array}{cc}1 & 0 \\
0 & -1\end{array}$ \\
\hline & $(2,3)$ & $\frac{1}{2}$ & $\begin{array}{ll}-1 & \sqrt{3} \\
\sqrt{3} & 1\end{array}$ \\
\hline & $(3,1)$ & $\frac{1}{2}$ & $\begin{array}{cc}-1 & -\sqrt{3} \\
-\sqrt{3} & 1\end{array}$ \\
\hline \multirow[t]{2}{*}[3]{} & (123) & $\frac{1}{2}$ & $\begin{array}{cc}-1 & \sqrt{3} \\
-\sqrt{3} & -1\end{array}$ \\
\hline & (132) & $\frac{1}{2}$ & $\begin{array}{ll}-1 & -\sqrt{3} \\
\sqrt{3} & -1\end{array}$ \\
\hline
\end{tabular}


which does not reveal anything. For $\mathbf{R}=\mathbf{0}$, however, (50) implies $\phi_{m}(-\mathbf{x})=$ $(-1)^{m} \phi_{m}(\mathbf{x})$ or in the short hand notation

$$
\phi_{m}(i-k)=(-1)^{m} \phi_{m}(k-i)
$$

with $m$ the orbital angular momentum of the pair solution, we obtain

$$
\begin{aligned}
\Phi^{s}=\frac{1}{\sqrt{6}} & \left\{\phi_{1}(2-3)\left[\phi_{2}(3-1) \phi_{3}(1-2)-(-1)^{M_{L}} \phi_{2}(1-2) \phi_{3}(3-1)\right]\right. \\
& +\phi_{2}(2-3)\left[\phi_{3}(3-1) \phi_{1}(1-2)-(-1)^{M_{L}} \phi_{3}(1-2) \phi_{1}(3-2)\right] \\
& \left.+\phi_{3}(2-3)\left[\phi_{1}(3-1) \phi_{2}(1-2)-(-1)^{M_{L}} \phi_{1}(1-2) \phi_{2}(3-1)\right]\right\}
\end{aligned}
$$

where $M_{L}=\sum_{i=1}^{3} m_{i}$ is the total orbital angular momentum. If we define a matrix of pair functions

$$
\mathbf{S}=\left[\begin{array}{lll}
\phi_{1}(2-3) & \phi_{1}(3-1) & \phi_{1}(1-2) \\
\phi_{2}(2-3) & \phi_{2}(3-1) & \phi_{2}(1-2) \\
\phi_{3}(2-3) & \phi_{3}(3-1) & \phi_{3}(1-2)
\end{array}\right]
$$

then our result can be written as a determinant or permanent] of pair functions

$$
\begin{aligned}
& \Phi^{s}=\frac{1}{\sqrt{6}} \operatorname{Det}(\mathbf{S}) \quad \text { for } M_{L}=\text { even } \\
& \Phi^{s}=\frac{1}{\sqrt{6}} \operatorname{Perm}(\mathbf{S}) \quad \text { for } M_{L}=\text { odd }
\end{aligned}
$$

From this fact we conclude the following important rule valid for $\mathbf{R}=\mathbf{0}$ :

If at least two pair functions agree, $M_{L}$ has to be odd.

Otherwise the determinant vanishes. This means in particular, that the total ground state cannot be built up from identical ground state pair functions with $m=0$, which would have the lowest energy without taking the Pauli principle into account. Therefore, the ground state consists of one pair with $m=1$ and two pairs with $m=0$ and has a total angular momentum $M_{L}=1$ in the strong correlation limit.

Observe the difference of these conclusions to the Pauli principle for oneelectron orbitals. If $\Phi$ were a product of functions which depend on one

\footnotetext{
${ }^{7} \mathrm{~A}$ permanent is similar to a determinant without the factors $(-1)^{p}$
} 
coordinate only (one-particle orbitals), which is equivalent to a Slater determinant for $\Phi^{s}$, in the quartet state all three spatial functions must be different, and the ground state would have a total angular momentum $M_{L}=$ $(m-1)+m+(m+1)=3 m$ where the integer $m$ depends on the strength of the magnetic field. This holds if $\tilde{\omega}$ is so large that no excited one-particle orbitals are involved, i.e. in the weak correlation regime. Indeed, for strong fields the simple rule that $M_{L}$ is a multiple of 3 is confirmed also by numerical calculations [1], [17], 近.

\section{Doublet $S=\frac{1}{2}$}

Because of $f=2$ there are two degenerate and orthogonal functions $\Psi_{i}$ which span a subspace. This level does not exist from symmetry reasons only if both functions vanish. We assume $\mathbf{R}=\mathbf{0}$ so that (90) holds and consider the two vectors $\boldsymbol{\Phi}_{i}^{s}$ one by one.

For $i=1$ and $M_{L}=$ even we obtain

$$
\begin{aligned}
& \boldsymbol{\Phi}_{1}^{s}=\frac{1}{\sqrt{3}}\left\{\left[\begin{array}{l}
1 \\
0
\end{array}\right] \phi_{3}(1-2) D_{12}(2-3,3-1)\right. \\
& +\frac{1}{2}\left[\begin{array}{c}
-1 \\
-\sqrt{3}
\end{array}\right] \quad \phi_{3}(2-3) \quad D_{12}(3-1,1-2) \\
& \left.+\frac{1}{2}\left[\begin{array}{l}
-1 \\
\sqrt{3}
\end{array}\right] \phi_{3}(3-1) D_{12}(1-2,2-3)\right\}
\end{aligned}
$$

where

$$
D_{12}(2-3,3-1)=\operatorname{Det}\left[\begin{array}{ll}
\phi_{1}(2-3) & \phi_{1}(3-1) \\
\phi_{2}(2-3) & \phi_{2}(3-1)
\end{array}\right]
$$

and the other determinants are defined analogously: the subscripts refer to the quantum numbers of the pair functions involved and the arguments define the arguments of the pair functions.

For $i=1$ and $M_{L}=o d d$ we obtain a similar formula as before but with the determinants in (94) replaced by permanents called $P_{12}$.

For $i=2$ and $M_{L}=$ odd the result is also similar but with different column vectors

$$
\boldsymbol{\Phi}_{2}^{s}=\frac{1}{\sqrt{3}}\left\{\left[\begin{array}{l}
0 \\
1
\end{array}\right] \phi_{3}(1-2) D_{12}(2-3,3-1)\right.
$$




$$
\begin{aligned}
& +\frac{1}{2}\left[\begin{array}{c}
\sqrt{3} \\
-1
\end{array}\right] \quad \phi_{3}(2-3) D_{12}(3-1,1-2) \\
& \left.+\frac{1}{2}\left[\begin{array}{c}
-\sqrt{3} \\
-1
\end{array}\right] \phi_{3}(3-1) \quad D_{12}(1-2,2-3) \quad\right\}
\end{aligned}
$$

where $D_{12}$ is defined as in (94).

For $i=2$ and $M_{L}=$ even the determinants in the last formula have to be replaced by permanents.

It is obvious that for $\phi_{1}=\phi_{2}\left(\neq \phi_{3}\right)$ the determinants $D_{12}$ vanish and consequently for $M_{L}=$ even, $\boldsymbol{\Phi}_{1}^{s}$ vanishes and for $M_{L}=o d d, \boldsymbol{\Phi}_{2}^{s}$ vanishes, but both do not vanish simultaneously. Therefore this state is allowed. However, if all three pair functions agree, also those functions vanish in which permanents occur, because the sum of the prefactors sum up to zero. Consequently, the Pauli-principle tells us that for $\mathbf{R}=\mathbf{0}$ in the doublet state: All three pair functions must not agree.

This is analogous to the one-particle model, where two orbitals may agree (if they occupy different spin states), but not all three of them. This means that, as for the quartet, the ground state consists of one pair with $m=1$ and two pairs with $m=0$ and it has a total angular momentum $M_{L}=1$. Thus, in our limit and for vanishing magnetic field, the quartet and the doublet ground state energy agree. This degeneracy is lifted by a magnetic field because of the Zemann energy.

\section{Acknoledgement}

I am indebted to H.Eschrig, W.Weller and their groups as well as J.K.Jain, W.Apel, and U.Zülicke for discussion and the Deutsche Forschungs- Gemeinschaft for financial funding. 


\section{References}

[1] S.M. Girvin, T. Jach, Phys. Rev. 28, 4506 (1983)

[2] U. Merkt, J. Huser, M. Wagner, Phys. Rev. B 43, 7320 (1991);

M. Wagner, U. Merkt, and A.V. Chaplik, Phys. Rev. B 45, 1951 (1992)

[3] P. A. Maksym, T. Chakraborty, Phys. Rev. Lett. 65, 108 (1990) and Phys. Rev. B 45, 1947 (1992);

P.A. Maksym, Physica B 184, 385 (1993)

[4] P. Hawrylak and D. Pfannkuche, Phys. Rev. Lett. 70, 485 (1993);

P. Hawrylak, Phys. Rev. Lett. 71, 3347 (1993)

[5] M. W. C. Dharma-wardana, J. Phys. Condens. Matter 7, 4095 (1995) ; and Phys. Rev. B 51, 1653 (1995)

[6] S.-R. E. Yang, A. H. MacDonald, and M. D. Johnson, Phys. Rev. Lett. 71, 3194 (1993); S. Mitra and A. H. MacDonald, Phys. Rev. B 48, 2005 (1993)

[7] R.B. Laughlin, Phys. Rev. 27, 3383 (1983);

R. B. Laughlin, in: The Quantum Hall Effect, Eds. R.E.Prange and S.M.Girvin, Springer Verlag, New York (1987).

[8] J. K. Jain, in: Perspectives in Quantum Hall Effects, Ed.s S. Das Sharma and A. Pinczuk, John Willey \& Sons,(1996)

[9] J. K. Jain and T.Kawamura, Europhys. Lett. 29, 321 (1995); R. K. Kamilla and J. K. Jain, Phys. Rev. B 52, 2798 (1995); T.Kawamura and J. K. Jain, J. Phys. Condens. Matter 8,2095 (1996)

[10] B.I. Halperin, in: Perspectives in Quantum Hall Effects, Ed.s S. Das Sharma and A. Pinczuk, John Willey \& Sons,( 1996)

[11] F. D. M. Haldane, in: The Quantum Hall Effect, Springer Verlag, New York (1987).

[12] A. H. MacDonald, in: Mesoscopic Quantum Physics, Proceedings of the Les Houches Summer School, Elsevier (1995) 
[13] M. Taut, J. Phys. A27, 1045 (1994) and erratum J.Phys.A27, 4723 (1994). Additionally, in formula (10) in the term containing $\frac{\partial}{\partial \alpha}$ a factor $\frac{1}{2}$ is missing, and on the r.h.s. of (19a) and (20a) $\tilde{\omega}$ must be replaced by $\tilde{\omega}_{r}$.

[14] This fact is unpublished, but it is completely analogous to the case of two electrons in an external oscillator potential in 3 dimensional space. See M.Taut, A.Ernst and H.Eschrig; J. Phys. B 31,2689 (1998)

[15] W. Y. Ruan et al., Phys. Rev. B 51, 7942 (1995)

[16] T. Seki, Y. Kuramoto, T. Nishino, J. Phys. Soc. Japan 65, 3945 (1996)

[17] P. A. Maksym, Phys. Rev. B 53, 10871 (1996)

[18] V. Fock, Z. Phys. 47, 446 (1928) (in German); C.G. Darwin, Proc. Cambridge Philos. Soc. 27, 86 (1930); R.B. Dingle, Proc. Roy. Soc. London A 211, 500 (1952)

[19] R. Pauncz, Spin Eigenfunctions, Plaenum Press, New York and London 1979 